\title{
A quantum genetic algorithm with quantum crossover and mutation operations
}

\author{
Akira SaiToh • Robabeh Rahimi • Mikio \\ Nakahara
}

Received: date / Accepted: date

\begin{abstract}
In the context of evolutionary quantum computing in the literal meaning, a quantum crossover operation has not been introduced so far. Here, we introduce a novel quantum genetic algorithm which has a quantum crossover procedure performing crossovers among all chromosomes in parallel for each generation. A complexity analysis shows that a quadratic speedup is achieved over its classical counterpart in the dominant factor of the run time to handle each generation.
\end{abstract}

Keywords Genetic algorithm · Quantum computing · Computational complexity

PACS 03.67.Ac $\cdot 87.23 .-\mathrm{n} \cdot 89.70 . \mathrm{Eg}$

\footnotetext{
A. SaiToh

Quantum Information Science Theory Group, National Institute of Informatics, 2-1-2 Hitotsubashi, Chiyoda, Tokyo 101-8430, Japan

R. Rahimi

Institute for Quantum Computing, University of Waterloo, 200 University Avenue West, Waterloo, Ontario N2L 3G1, Canada

M. Nakahara

Department of Physics, Kinki University, 3-4-1 Kowakae, Higashi-Osaka, Osaka 577-8502, Japan

A. SaiToh · M. Nakahara

Research Center for Quantum Computing, Interdisciplinary Graduate School of Science and Engineering, Kinki University, 3-4-1 Kowakae, Higashi-Osaka, Osaka 577-8502, Japan

Present Address:

A. SaiToh

Department of Computer Science and Engineering, Toyohashi University of Technology, 1-1

Hibarigaoka, Tenpaku-cho, Toyohashi, Aichi 441-8580, Japan

E-mail: saitoh@sqcs.org
} 


\section{Introduction}

Continuous development has been performed on genetic algorithms [19, 11, 32 . Along with the development of quantum computing [15,37, quantum-inspired classical algorithms for evolutionary computing have been developed [36, 16. 17, 18, 35, 34, 6, 24, 33. (see also a review [54] and references therein). In addition, classical genetic algorithms to evolve quantum circuits have also been studied by several authors $[52,44,2,45,53,38,25,22,43,23,29,26,30,7,46$ ] (see also review articles 10,9). These algorithms are, however, designed to work on classical computers. Quantum genetic algorithms (QGAs), in its literal meaning, nonetheless, have gathered comparably little attention and a few works [40, 49, 27, 50, 28, have been performed so far. Evolutionary computing on quantum architectures will achieve more attention if there is a scenario to establish significant improvement over classical counterparts. Indeed, Malossini et al. 27,28, claimed that the computational complexity of an evolutionary step between generations is exponentially fast in their quantum algorithm in comparison to a classical one. It seems, however, that they overlooked the complexity of index-to-string conversion circuits or, otherwise, overlooked the circuit complexity of a variant of the inversion-about-average operation. Their claim is thus misleading as we will discuss in Sect. 2, Recently, Johannsen et al. 20 applied quantum search algorithms to several optimization problems in a certain context of evolutionary computing. Nevertheless, it is obscure how much cost is spent for the internal quantum circuit of a variant of the inversion-about-average operation used for amplitude amplification (namely, the operation denoted as $\mathcal{A S} \mathrm{S}_{0}^{\phi} \mathcal{A}^{-1}$ in the convention of Ref. [4]) in their approach.

Let us briefly summarize the conventional approach of QGAs and its problem. Here, we omit discussions on incomplete or physically unfeasible works on QGAs, which were summarized by Sofge in Ref. [41]. The aim of a genetic algorithm is, in most cases, to find an individual (typically, an input string) with a very high fitness value for a given problem. It starts with $O\left(|A|^{w}\right)$ initial individuals where $|A|$ is the size of the alphabet $A$ and $w$ is the length of a schema expected to be a building block for a given problem. Let us restrict the problem by representing each individual as a chromosome encoded as a binary string with the length $n$. We need $\sim N=2^{n}$ initial chromosomes for the worst case. With a quantum register, one may use $n$ qubits to make a superposition of $N$ chromosomes. We regard the probability to find a chromosome on the computational basis as its (normalized) population.

First we briefly overview the selection strategies in conventional QGAs. In short, a selection is an operation to enhance populations of individuals with high fitness values and to decrease those with low fitness values. In quantum computing, it is natural to utilize variants of Grover's algorithm [12] for this purpose. In fact, a variant of the Grover search for a priori unknown number $r$ of solutions (the Grover-BBHT search) $[3$ and that for finding the maximum 8,1 are the essential parts of the QGAs developed in Refs. 49, 27, 50, 28, The query complexity $O(\sqrt{N / r})$ for a variant of the Grover search dominates 
the total complexity in the QGA of Udrescu et al. 49,50. For the QGA of Malossini et al. [27, 28, there is a different factor to consume time. As mentioned, this will be discussed in detail in Sect. 2 .

Second we overview the strategies for crossovers and mutations in conventional QGAs. In a short explanation of these terms, a crossover is an operation to exchange substrings of two chromosomes and a mutation is an operation to flip certain bits of a chromosome. These are effective operations to enlarge the search space. So far, quantum crossover operations have not been developed. Malossini et al. [27,28, used classical crossover and mutation operations; Udrescu et al. 49,50 did not use crossover and mutation operations. Johannsen et al. 20 introduced quantum mutation operations in an application of quantum amplitude amplification [4 to some optimization problems; nevertheless, crossovers were not used. It is, in fact, in general difficult to manipulate populations of quantum states for handling crossovers if we imitate the classical way in a straightforward manner (See also Ref. 10 which explained the difficulty in a slightly different manner). Picking-up two particular individuals and making a crossover costs $O(n)$ quantum gates if we use $O(n)$ ancillary qubits. Since there are many possible pairs for a crossover at each generation, there is no speedup over a classical crossover. More specifically speaking, this approach needs to look up classical data of chromosomes to specify a pair of chromosomes. Thus there cannot be any speedup. In addition, this requires an exponentially large classical memory in comparison to the size of a quantum register. We will show a different approach to handle crossovers in our algorithm in Sect. 3.

As another direction for developing quantum evolutionary computing, which we do not pursue in this contribution, one may use so-called quantum fixedpoint search algorithms 14,48. They increase the population of a superposition of target chromosomes by iterative applications of unitary amplitudeamplification operations. Unlike the original Grover search, there is no drawback in an excessive iteration; the probability of finding a target grows monotonically as $1-\epsilon^{2 t+1}$ where $\epsilon$ is the probability of finding a non-target chromosome and $t$ is the number of queries. The query complexity of this approach is, however, as large as that of the exhaustive search: When the number $r$ of targets is very small in comparison to $N$, we have $\epsilon^{2 t+1}=(1-r / N)^{2 t+1} \simeq$ $1-(2 t+1) r / N$, which implies that $O(N / r)$ queries are required to achieve a sufficiently small error probability, say $1 / 2$. Thus for $r \ll N$, Grover's algorithm should be chosen instead of fixed-point quantum search algorithms. In addition, the asymptotic optimality of $\epsilon^{2 t+1}$ as an error reduction speed was proved [5 for fixed-point quantum search methods. It is also known that the query complexity of the Grover search is optimal [3] in general as a unitary process for unsorted search. Therefore, it is unlikely 11 that a fixed-point

1 There is another drawback in the use of a quantum fixed-point search. It requires phase shift operations $\widetilde{U}_{\mathrm{s}}=1-\left(1-e^{i \pi / 3}\right) \sum_{l}\left|\varsigma_{l}\right\rangle\left\langle\varsigma_{l}\right|$ and $\widetilde{U}_{\mathrm{t}}=1-\left(1-e^{i \pi / 3}\right)\left|\tau_{m}\right\rangle\left\langle\tau_{m}\right|$ with source states $\left|\varsigma_{l}\right\rangle$ and target states $\left|\tau_{m}\right\rangle$ ( $l$ and $m$ are labels) in addition to another appropriate unitary operation [14]. One may alternatively use $\hat{U}_{\mathrm{s}}=1-\left(1-e^{i \pi / 3}\right)|\mathcal{S}\rangle\langle\mathcal{S}|$ and $\hat{U}_{\mathrm{t}}=1-\left(1-e^{i \pi / 3}\right)|\mathcal{T}\rangle\langle\mathcal{T}|$ where $|\mathcal{S}\rangle$ is an equally-weighted superposition of $\left|\varsigma_{l}\right\rangle$ and $|\mathcal{T}\rangle$ is 
quantum search is effectively used in an evolutionary computing instead of the standard Grover search and its variants.

In this contribution, we propose a quantum genetic algorithm that involves a quantum crossover and a quantum mutation. It uses the quantum search for finding the maximum 8, 1 for the selection procedure. Although this selection strategy looks similar to those of conventional QGAs [49,27,50,28, it is different in the point that we use tailored inversion operations for the quantum search. Our algorithm achieves a quadratic speedup over its classical counterpart in the dominant factor of the run time to update each generation as an algorithm involving all possible crossovers for a chosen crossing site.

This paper is organized in the following way. Section 2 discusses on the algorithm of Malossini et al. 27,28. Section 3 describes our algorithm. The procedures of the crossover, the mutation and the selection are developed in the standard quantum circuit model. Computational complexities of the algorithm are evaluated in Sect. 4. The obtained results are discussed in Sect. 5 and summarized in Sect. 6.

\section{A conventional quantum genetic algorithm}

Here we discuss the conventional quantum genetic algorithm proposed by Malossini et al. 27,28. There is another conventional algorithm proposed by Udrescu et al. 49, 50, which is not introduced here. The main difference is that Udrescu et al. did not employ crossovers and mutations while Malossini et al. employed them as classical operations.

The problem we consider is given as follows.

Problem: Suppose there are $N$ individuals with indices $0, \ldots, N-1$. There is a fitness function $f:\{0, \ldots, N-1\} \rightarrow[0,1]$. Find one of the individuals with sufficiently large fitness values.

The algorithm of Malossini et al. is described in Fig. 1 (Algorithm 1). They analyzed their algorithm and claimed [27,28, that the selection procedure internally requires a very small number of oracle calls in contrast to $O(\widetilde{N} \log \tilde{N})$ oracle calls required by a classical selection procedure, where $\widetilde{N}$ is the number of individuals in a generation. In short, their claim was that the number of the Grover iterations in step (b) (of Fig. 1) was $O(1)$.

Their complexity analysis was, however, misleading. A more accurate description of the complexity is given in the following way. There are two possible cases we should consider: (A) $\widetilde{N}$ is approximately equal to $N$; (B) $\widetilde{N} \lesssim N$.

Let us consider the case (A). We can preset the index $x$ to represent the $x$ th individual in a good approximation thanks to $\widetilde{N} \simeq N$. Then the given fitness function can be used as it is, without index conversion. This indicates that we

an equally-weighted superposition of $\left|\tau_{m}\right\rangle$. (This kind of alternative operation for a quantum search was used in Ref. 47.) The problem is that $\varsigma_{l}$ 's are highly-random nonconsecutive chromosomes in the context of evolutionary computing. There is no known way to construct $\widetilde{U}_{\text {s }}$ or $\hat{U}_{\mathrm{s}}$ within poly $(\log \widetilde{N})$ cost in such a case, where $\widetilde{N}$ is the number of $\varsigma_{l}$ 's. 
Algorithm 1 (Malossini et al. 27 28])

Main Procedure:

Start from a generation consisting of $\tilde{N} \leq N$ individuals.

Repeat:

1. Use the Quantum Selection Procedure to get one index. Do the same procedure to get another.

2. Classically make a crossover for the two individuals corresponding to the indices obtained in 1. Classically make a proper mutation.

3. Classically replace randomly chosen two individuals with the two offsprings obtained in 2 .

Quantum Selection Procedure:

(i) Choose randomly an index $y$ among $\widetilde{N}$ possible ones in the present generation and compute its fitness $f(y)$. Set the threshold $F_{y} \longleftarrow f(y)$.

(ii) Perform $\tau$ times:

(a) Initialize the quantum registers to $\frac{1}{\sqrt{\widetilde{N}}} \sum_{x}|x\rangle|y\rangle$, where $x$ 's are the indices of the present generation.

(b) Apply the Grover-BBHT search [3], where we internally use an oracle that inverts the signs of the marked states, namely states $|x\rangle$ such that $f(x) \geq F_{y}$. This enhances the amplitudes of marked states after its Grover

(c) Measure the left register in the computational basis and get the new index $y^{\prime}$. If $f\left(y^{\prime}\right)>F_{y}$, then $y \leftarrow y^{\prime}$ and $F_{y} \leftarrow f\left(y^{\prime}\right)$.

(iii) Return the index $y$.

Fig. 1 Description of Malossini et al.'s algorithm.

use nothing but the standard Grover-BBHT search for $N$ indices. As $\widetilde{N} \simeq N$, the quantum selection procedure is called only once or a very small number of times. Suppose there are $r$ nearly-optimal individuals that are acceptable as solutions for a given problem. Inside the quantum selection procedure, as the selection goes on in (ii), the oracle used in step (b) has less marked states. The number of marked states converges to $r$. Therefore, the query complexity of a single step in a very later selection stage is $O(\sqrt{\widetilde{N} / r})=O(\sqrt{N / r})$. This is the accurate description of the query complexity for the present case and it is the same as that Udrescu et al. gave for their algorithm [49,50. On average, each time of the repetition in (ii) virtually extinguishes a half of the individuals by increasing the threshold. Thus, by considering the sum of the geometric series $\sqrt{N /\left(2^{i} r\right)}(i=0,1,2, \ldots)$, the average total query complexity is found to be $O(\sqrt{N / r})$.

Let us now consider the case (B). In this case, the quantum selection procedure is called several (or more) times. The important fact is that the chromosomes of individuals in a generation are not consecutive binary strings. The generation contains $\widetilde{N}$ binary strings out of $N$ possible ones. There are two options to handle them: (B-1) consecutive integers are assigned as pointers to nonconsecutive chromosomes; (B-2) nonconsecutive chromosomes are used as they are.

(B-1): In this case, the index $x$ is a pointer to the chromosome $\kappa_{x}$ for which the fitness function works appropriately. (In short, this is a workaround for the fact that the original Grover-BBHT search should start from a uniform superposition of consecutive indices while the generation consists of noncon- 
secutive chromosomes.) Therefore, for the superposition $\left|\psi_{0}\right\rangle=\frac{1}{\sqrt{\widetilde{N}}} \sum_{x}|x\rangle|y\rangle$, the fitness function cannot be applied directly. We need a conversion circuit to interpret $x$ as $\kappa_{x}$. Then the fitness function is applied and it returns $f\left(\kappa_{x}\right)$. Each conversion should involve $O(\log N)$ CCNOT gates. Thus, the total circuit depth for the index conversions is $O(\widetilde{N} \log N)$. As a result, a single oracle call accompanies the additional time complexity $O(\widetilde{N} \log N)$. Indeed, the query complexity is small if $\widetilde{N}$ is much smaller than $N$. However, the cost of index conversions hinders speedup over a classical selection procedure.

(B-2): In this case, the state $|x\rangle$ in the superposition $\left|\psi_{0}\right\rangle=\frac{1}{\sqrt{\widetilde{N}}} \sum_{x}|x\rangle|y\rangle$ keeps the actual chromosome $x$ as an index. One should use the Grover-search routine somehow without index conversions by choosing $\left|\psi_{0}\right\rangle$ as its initial state. Indeed, this is possible 4] in the case where one can provide the operation $L=I-2\left|\psi_{0}\right\rangle\left\langle\psi_{0}\right|$ as the inversion-about-average operation instead of the standard one for the original Grover search. Nevertheless, $L$ should be generated by sandwiching $I-2|0\rangle\langle 0|$ with $U_{(\text {a) }}$ and $U_{(\text {a) }}^{\dagger}$ in the present context, where the unitary operation $U_{(\text {a) }}$ corresponds to the initialization step (a). Thus the circuit complexity of $L$ is $O(\tilde{N} \log N)$ (see, e.g., Ref. [51]). (There is a little confusing result by Soklakov and Schack [42; they showed that a state preparation, namely an initialization, is possibly performed within polynomial $\operatorname{cost}$ in $\log N$ for some cases. Nevertheless, considering the internal cost of the special oracle they use, their method spends $O(\widetilde{N} \operatorname{poly}(\log N))$ time for general cases including the present case where the parent set consists of random indices.) As a consequence, an expensive circuit for $L$ should be used subsequent to every query. Obviously, the time complexity in this case is as large as the one in case (B-1).

One may also think of replacing the Grover-search routine with the generalized Grover search for nonconsecutive integer sets [13. Nevertheless, it is required to find an appropriate unitary transformation replacing the standard Hadamard transformation $H \otimes \cdots \otimes H$. There is no known way to find it efficiently when a random integer set is given and target integers are unknown in advance.

There is, in fact, a way to reduce the circuit complexity of $L$ introduced in (B-2) if the algorithm is modified so that it uses an efficient pseudo-randomizer (or, pseudo-scrambler) circuit instead of directly using a random number generator. Our algorithm introduced in the next section takes this approach.

In addition to the above discussions, we should mention that Algorithm 1 has another problem: it takes $O(\widetilde{N} \log N)$ space to keep a generation in a classical memory. This is usually quite larger than the $O(\log N)$ space that is enough for quantum search (neglecting the space internally used by an oracle circuit). Our algorithm is designed not to face this problem. 


\section{Algorithm with quantum crossover and quantum mutation}

As we have seen in the previous sections, conventional quantum genetic algorithms were not designed to achieve quantum speedup in their selection procedures in case a generation consists of nonconsecutive chromosomes. In addition, a quantum crossover operation has not been developed so far. Here, we propose an algorithm using a quantum crossover and a quantum mutation. We use a variant of the Grover search with tailored subroutines for the selection procedure, whose query complexity is the main factor of the total time complexity. We achieve a quadratic speedup over a classical counterpart in a dominant factor of time complexity as a genetic algorithm with crossovers among all parents. It is novel in the sense that a simultaneous crossover using a superposition is achieved. The speedup partly relies on an efficient internal structure of a pseudo-randomizer circuit, which will be explained in Appendix A.

\subsection{Algorithm flow}

We introduce our algorithm in Fig. 2 (Algorithm 2). In this algorithm, the quantum register is accessible from its subroutines as a kind of global variables. The procedures called inside the algorithm are described in corresponding subsections 3.23 .5 .

\subsection{Preparing the initial state of a quantum register}

In this subsection, we define the procedure init_reg $(R, z)$.

This procedure is intended to prepare a superposition corresponding to the generation given as a set $X=\{R(q)\}_{q} \cup\{z\}$ with $q=0_{0} \cdots 0_{c-2} 1_{c-1}, \ldots, 1_{0} \cdots 1_{c-1}$ (thus $\sharp X=\widetilde{N}=2^{c}$ ). The procedure starts with the quantum state $|0\rangle_{\mathrm{a}}^{\otimes c}|0\rangle^{\otimes n}$. The desired superposition is $|\varphi\rangle=\frac{1}{\sqrt{\widetilde{N}}} \sum_{x \in X}\left|a_{x}\right\rangle_{\mathrm{a}}|x\rangle$ with $a_{x}$ the address pointing $x$. We opt to use consecutive addresses $0, \ldots, 2^{c}-1$. The procedure is now defined in Fig. 3. The order of time complexity of this procedure equals to the internal circuit complexity $\operatorname{poly}(\mathrm{cn})$ of the pseudo-randomizer $R$. An explicit example to construct $R$ as a quantum circuit is given in Appendix A.

\subsection{Crossover}

Here, we construct a quantum crossover procedure quantum_crossover $(l)$. It is a 1-point crossover acting on the chromosomes simultaneously.

Recall that the original generation is given as a set $X$ of $n$-bit integers $x$ with $\sharp X=\tilde{N}$. Each $x$ has its left side $x^{\text {left }}$ and its right side $x^{\text {right }}$ separated by the crossing site. The crossing site is placed between the $(l-1)$ th and the $l$ th qubits as specified by the parameter. Hence, the bit length of $x^{\text {left }}$ is $l$. 


\section{Algorithm 2}

Consider a threshold $f_{\text {th }}$ for fitness values, which is considered to be sufficiently large. REPEAT 1.-9.:

1. Construct a pseudo-randomizer $R$ that maps a $c$-bit string to a pseudo-random $n$-bit string. It should be implemented as a circuit whose input and output are integer couples $(a, 0)$ and $(a, R(a))$, respectively, where $a$ is a $c$-bit integer. The circuit should consist of poly $(c n)$ elementary reversible logic gates. We require $R$ to regard $0_{0} \cdots 0_{c-1}$ as an exception and map it to $0_{0} \cdots 0_{n-1}$. We also require $\widetilde{N}=2^{c} \succsim N=2^{n}$. Once constructed, $R$ is fixed until next $t$. An explicit example to construct $R$ is given in Appendix A.

2. IF $t=0$ THEN generate a random $c$-bit string $\gamma$ and set $z \longleftarrow R(\gamma)$ (otherwise, $z$ is the best chromosome found in the $(t-1)$ th trial) ENDIF

3. Generate a random $c$-bit string $\gamma^{\prime}$ and set $u \longleftarrow R\left(\gamma^{\prime}\right)$.

4. CALL init_reg $(R, z)$ defined in Sect. 3.2 twice to make two identical quantum states that are both $|\varphi\rangle=\frac{1}{\sqrt{\widetilde{N}}} \sum_{x \in X}\left|a_{x}\right\rangle_{\mathrm{a}}|x\rangle$ where $a_{x}$ is the address pointing to $x \in X ; X=\{R(q)\}_{q} \cup\{z\}$ with $q=0_{0} \cdots 0_{c-2} 1_{c-1}, \ldots, 1_{0} \cdots 1_{c-1}$; subscript "a" stands for the address portion. We write the entire unitary operation of this procedure as $U_{\text {init. }}$ That is, $|\varphi\rangle^{\otimes 2}=U_{\text {init }}\left(|0\rangle_{\mathrm{a}}|0\rangle\right)^{\otimes 2}$.

5. CALL quantum_crossover $(l)$ defined in Sect. 3.3 for the current quantum register, which is a 1-point crossover with the crossing site, chosen at one's convenience, placed between the $(l-1)$ th and the $l$ th bits of a chromosome. All possible crossovers for this crossing site are performed simultaneously. This procedure is an identity map as a quantum operation acting on $|\varphi\rangle^{\otimes 2}$.

6. Apply the quantum mutation (Sect. 3.4) to the current quantum register. We write the entire unitary operation of this procedure as $U_{\text {mut }}$. Apply the same mutation classically to $u$.

7. CALL quantum_selection $\left(U_{\text {init }}, U_{\text {mut }}, u\right)$ defined in Sect. 3.5 for the current quantum register and obtain the output chromosome $z$.

8. IF $f(z) \geq f_{\text {th }}$ THEN RETURN $z$ and EXIT ENDIF

9. $t \longleftarrow t+1$. Refresh the quantum register.

Fig. 2 Description of our algorithm.

PROCEDURE init_reg $(R, z)$ :

(i) Make a superposition $\frac{1}{\sqrt{\sharp X}} \sum_{j=0}^{2^{c}-1}|j\rangle_{\mathrm{a}}|0 \cdots 0\rangle$ by applying $H^{\otimes c} \otimes I^{\otimes n}$ to $|0\rangle_{\mathrm{a}}^{\otimes c}|0\rangle^{\otimes n}$.

(ii) Apply the pseudo-randomizer $R$ implemented as a unitary operation mapping $|j\rangle_{\mathrm{a}}|0\rangle$ to $|j\rangle_{\mathrm{a}}\left|x_{j}\right\rangle$ with $x_{j}$ an $n$-bit pseudo-random number for $j=1, \ldots, 2^{c}-1$. By assumption, $|0\rangle_{\mathrm{a}}|0\rangle$ is mapped to $|0\rangle_{\mathrm{a}}|0\rangle$.

(iii) Apply a $0_{0} \cdots 0_{c-1}$-controlled $X^{z_{0}} \otimes \cdots \otimes X^{z_{n-1}}$ to map $|0\rangle_{\mathrm{a}}|0\rangle$ to $|0\rangle_{\mathrm{a}}|z\rangle$, where $z_{k}$ is the $k$ th bit of $z(k=0, \ldots, n-1)$.

(iv) RETURN the current state, namely $|\varphi\rangle$.

Fig. 3 Description of procedure init_reg $(R, z)$.

With the procedure, we generate a superposition of all the children that are combinations of $x^{\text {left's }}$ and $x^{\text {right's }}$ together with their parents with the same weight as children.

The state of the quantum register in the beginning of this procedure is

$$
|\varphi\rangle^{\otimes 2}=\left(\frac{1}{\sqrt{\sharp X}} \sum_{x \in X}\left|a_{x}\right\rangle_{\mathrm{a}}\left|x^{\text {left }}\right\rangle\left|x^{\text {right }}\right\rangle\right) \otimes\left(\frac{1}{\sqrt{\sharp X}} \sum_{x^{\prime} \in X}\left|a_{x^{\prime}}\right\rangle_{\mathrm{a}}\left|x^{\prime \text { left }}\right\rangle\left|x^{\prime \text { right }}\right\rangle\right) .
$$


This state has the components $\left|x^{\text {left }}\right\rangle\left|x^{\text {right }}\right\rangle\left|x^{\text {left }}\right\rangle\left|x^{\prime \text { right }}\right\rangle$ besides the addresses. We relabel the qubits so that the middle portion $\left|x^{\text {right }}\right\rangle\left|x^{\prime l e f t}\right\rangle$ is put aside from our minds. Let us conceal them by denoting as $\left|*_{x x^{\prime}}\right\rangle$. In addition, we denote the main portion $\left|x^{\text {left }}{x^{\prime r i g h t}}^{\text {rith }}\right\rangle$ withe subscript "main". The state $|\varphi\rangle^{\otimes 2}$ with the new qubit labels is written as

$$
\frac{1}{\sharp X} \sum_{x \in X} \sum_{x^{\prime} \in X}\left|a_{x} a_{x^{\prime}}\right\rangle_{\mathrm{a}}\left|x^{\text {left }} x^{\text {right }}\right\rangle_{\text {main }}\left|*_{x x^{\prime}}\right\rangle .
$$

We have at most $(\sharp X)^{2}$ distinct chromosomes in this state. In this way, all possible crossovers are performed at once by the relabelling. The resultant state is a superposition of all of the children together with their parents. (The parents are involved because the values of $x$ and $x^{\prime}$ may coincide.) This is desirable as a crossover because sometimes some parents have higher fitness values than any child.

The procedure described above is formally written as shown in Fig. 4 .

PROCEDURE quantum_crossover $(l)$ :

(i) We have the quantum register in the state $|\varphi\rangle^{\otimes 2}$. The original labels of its qubits are $0, \ldots, 2 c+2 n-1$. We relabel them as $\underbrace{0, \ldots, c-1}_{c}, \underbrace{2 c, \ldots, 2 c+l-1}_{l}$,

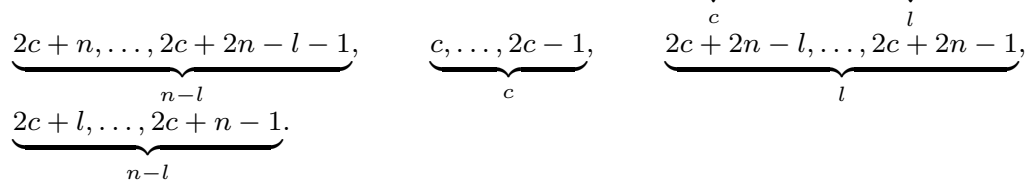

(ii) RETURN

Fig. 4 Description of procedure quantum_crossover $(l)$.

As is obvious, this procedure is an identity map as a quantum operation. Once the crossover process is completed, one may use a mutation as an option. This is going to be explained in the next subsection.

\subsection{Mutation}

In classical genetic algorithms, randomly selected chromosomes are affected by a mutation, which is typically certain bit flips acting on randomly-chosen places. Here, we consider the mutation procedure described in Fig. 5. Although it is written as a classical routine, it can be trivially interpreted as a quantum circuit. As a quantum circuit, this mutation procedure tmp_mut is realized by a multiple-bit controlled multiple-bit NOT gate. (In the example mentioned in Fig. 5, the gate is "0-controlled 1-controlled 1-controlled 0-controlled 1-controlled NOT NOT" with control bits specified by the first template and the target bits specified by the second template.) The gate 
PROCEDURE tmp_mut:

(i) Let us randomly generate the first template like $* * * 0 * 1 * * * 1 * * 0 * * * * 1 *$, which specifies a schema to mutate. Using this template, we pick up chromosomes with specified bits like $0,1,1,0,1$ in the specified places.

(ii) We also use the second template like $* \mathrm{X} * * * * * * * * * * * * * \mathrm{X} * * *$ in which $\mathrm{X}$ 's can be placed only on the places where *'s (namely, "don't care" symbols) are placed in the first template. Using this template, we apply the bit flip $\mathrm{X}$ to the specified places of the chromosomes picked up in (i).

(iii) RETURN

Note: Technically, we often wish to avoid a mutation for the best chromosome $z$ found so far by the present time step. This is realized by choosing the first template so that this does not happen.

Fig. 5 Description of procedure tmp_mut.

acts on the portion $\left|x^{\text {left }} x^{\prime \text { right }}\right\rangle_{\text {main }}$; the addresses $a_{x}$ and $a_{x^{\prime}}$ are untouched. Therefore, the resultant state can be written as

$$
|\widetilde{\varphi}\rangle=\frac{1}{\sharp X} \sum_{x \in X} \sum_{x^{\prime} \in X}\left|a_{x} a_{x^{\prime}}\right\rangle_{\mathrm{a}}\left|\widetilde{x}^{\mathrm{left}}{\widetilde{x^{\prime}}}^{\text {right }}\right\rangle_{\text {main }}\left|*_{x x^{\prime}}\right\rangle,
$$

where $\left(\widetilde{x}^{\text {left }}{\widetilde{x^{\prime}}}^{\text {right }}\right)$ 's are the chromosomes after the mutation process.

The next step is to apply a natural selection to the chromosomes living in the superposition $|\widetilde{\varphi}\rangle$.

\subsection{Selection}

In this subsection, we introduce our selection procedure. It is intended to find a chromosome having the maximum fitness among $\left(\widetilde{x}^{\text {left }}{\widetilde{x^{\prime}}}^{\text {right }}\right)$ 's. It utilizes the quantum search for finding the maximum [8, 1. As we have mentioned, conventional QGAs [49,27,50,28, have similar selection strategies. The difference from them is that we use tailored inversion operations for the quantum search.

Our selection procedure is called with three arguments: $U_{\text {init }}, U_{\text {mut }}$ and $u$. We have the state (10) at the beginning of this procedure. The procedure is now defined in Fig. 6. Here in the text, we do not repeat its description. It should be noted that, in the procedure, we set $k_{\text {term }}=\eta \times\left\lceil(45 / 2) \widetilde{N}+(28 / 5)\left(\log _{2} \tilde{N}\right)^{2}\right\rceil$ with integer constant $\eta \geq 1$.

Let us give an explanation about $k_{\text {term }}$, namely, the number of iterations. The defined procedure is the same as the well-known quantum search algorithm for finding the maximum 8, 1 except for the definitions of the inversion operation for targets and the inversion-about-average operation. In other words, we perform the quantum search for finding the maximum in the subspace $\operatorname{span}\left\{\left|a_{x} a_{x^{\prime}}\right\rangle_{\mathrm{a}}\left|\widetilde{x}^{\text {left }}{\widetilde{x^{\prime}}}^{\text {right }}\right\rangle_{\text {main }}\left|*_{x x^{\prime}}\right\rangle\right\}$. As proved by Dürr and Høyer [8], the probability for the output to be the maximum is at least $1 / 2$ if the number of iterations is $\left\lceil(45 / 2) \sqrt{M}+(7 / 5)\left(\log _{2} M\right)^{2}\right\rceil$ with $M$ the number of indices. In the present context, we have $M=\widetilde{N}^{2}$ since there are $\widetilde{N}^{2}$ distinct addresses. 
PROCEDURE quantum_selection $\left(U_{\text {init }}, U_{\text {mut }}, u\right)$ :

FOR $k \longleftarrow 0$ TO $k_{\text {term }}-1$ :

(i) Set

$$
U_{1}=I \otimes I \otimes I-2 \sum_{f(y) \geq f(u)} I \otimes|y\rangle\langle y| \otimes I
$$

where the left and the right $I$ 's act on the address states $\left|a_{x} a_{x^{\prime}}\right\rangle$ and the states $\left|*_{x x^{\prime}}\right\rangle$, respectively. This is the oracle function that can be implemented as follows. First, we attach ancillary qubits as blocks (I) and (II) in the state $|0 \cdots 0\rangle_{(\mathrm{I})}|-\rangle_{(\mathrm{II})}$ with $|-\rangle=(|0\rangle-|1\rangle) / \sqrt{2}$. For each $x x^{\prime}, f\left(\widetilde{x}^{\text {left }}{\widetilde{x^{\prime}}}^{\text {right }}\right)$ is set as a value of the block (I) by using $f$ implemented as a quantum circuit. Let us write this operation as $U_{f}$. Then we compare the block (I) with $f(u)$; we flip the qubit (II) if $f\left(\widetilde{x}^{\text {left }}{\widetilde{x^{\prime}}}^{\text {right }}\right) \geq f(u)$. We apply $U_{f}^{\dagger}$ (this disentangles the ancillary qubits from the main register) and remove the ancillary qubits.

(ii) Set

$$
U_{2}=I \otimes I \otimes I-2|\widetilde{\varphi}\rangle\langle\widetilde{\varphi}| .
$$

This operation is composed in the following way.

$$
U_{2}=U_{\text {mut }} U_{\text {init }}\left[I-2\left(|0\rangle^{\otimes c}|0\rangle^{\otimes n}\left\langle\left. 0\right|^{\otimes c}\left\langle\left. 0\right|^{\otimes n}\right)^{\otimes 2}\right] U_{\text {init }}^{\dagger} U_{\text {mut }}^{\dagger},\right.\right.
$$

where we also relabel qubits according to quantum_crossover $(l)$.

(iii) Apply the Grover-BBHT search 3 for which we use $U_{1}$ and $U_{2}$ instead of the standard operations, namely, the inversion operation for targets and the inversion-about-average operation, respectively. As for the starting state of the search, we use $|\widetilde{\varphi}\rangle$.

(iv) Measure the main register and obtain the chromosome $u^{\prime}$.

IF $f\left(u^{\prime}\right)>f(u)$ THEN $u \longleftarrow u^{\prime}$ ENDIF

$$
\text { ENDTFOR }
$$

Fig. 6 Description of procedure quantum_selection $\left(U_{\text {init }}, U_{\text {mut }}, u\right)$.

Therefore, after $k_{\text {term }}$ iterations, we find the output chromosome having the maximum fitness among $\left(\widetilde{x}^{\text {left }}{\widetilde{x^{\prime}}}^{\text {right }}\right)$ 's, with the probability at least $1-(1 / 2)^{\eta}$. By setting $\eta$ sufficiently large, say, around 16 to 24 , we have the desired output with almost certainty.

\section{Computational cost}

We are going to evaluate the computational cost of each process of Algorithm 2 (Fig. 2) in Sect. 4.1. The total computational cost to handle a generation will be derived in Sect. 4.2 and compared with that of classical counterpart in Sect. 4.3 .

\subsection{Costs of each procedure}

For a single call of each procedure, the costs are evaluated as follows.

Cost of the initialization The procedure to prepare the initial state, described in Sect. 3.2, uses poly $(c n)=\operatorname{poly}(\log \widetilde{N} \log N)$ elementary quantum gates. 
Cost of the crossover The crossover described in Sect. 3.3 makes use of two identical $(c+n)$-qubit states. This procedure does not use any quantum operation but relabels qubits. This takes $O(\log N)$ time.

Cost of the mutation The mutation described in Sect. 3.4 involves a single gate that looks like, say,

$$
\begin{aligned}
& *-\mathrm{NOT}-*-\mathrm{C}_{0}-*-\mathrm{C}_{1}-*-*-*-\mathrm{C}_{1}-*-* \\
& -\mathrm{C}_{0}-*-*-\mathrm{NOT}-*-\mathrm{C}_{1}-*
\end{aligned}
$$

with multiple control bits (0-control $\mathrm{C}_{0}$ 's and 1-control $\mathrm{C}_{1}$ 's) and multiple NOT gates placed according to the corresponding templates, where symbol * stands for an untouched qubit. Such a gate can be realized by $O(\log N)$ elementary quantum gates with $O(\log N)$ ancillary qubits.

Cost of the selection The selection described in Sect. 3.5 consumes $O(\widetilde{N})$ queries to find the best chromosome in the set of at most $\widetilde{N}^{2}$ chromosomes. Each query accompanies the operation $U_{2}$ that invokes $U_{\text {init }}$ and $U_{\text {mut }}$, and also their inverse operations. It is easy to find that the internal cost of $U_{2}$ is poly $(c n)$ according to the costs for $U_{\text {init }}$ and $U_{\text {mut }}$. In addition, the cost to prepare the starting state is poly $(c n)$. We may also mention that, the internal cost of the fitness function is a certain small factor, typically poly $(n)$, as conventionally assumed [11. Therefore, the circuit complexity of the procedure is $O(\widetilde{N} \operatorname{poly}(\log \widetilde{N} \log N))$. (We assume that the circuit depth is on the order of the circuit size, namely, the number of elementary quantum gates.) As for space, we use poly $(\log N)$ qubits in total, considering a typical fitness function consuming $\operatorname{poly}(n)$ space. When the fitness function is designed to use $O(n)$ space, $O(\log N)$ qubits are enough, although we do not assume this case for evaluating the space complexity.

\subsection{Total cost}

Comparing the costs of the four procedures, the dominant cost is the circuit complexity for the selection procedure. Therefore, we find that our algorithm uses

$$
O(\widetilde{N} \operatorname{poly}(\log \tilde{N} \log N))
$$

elementary quantum gates for each $t$. This is the time complexity of our algorithm for handling each generation. It is quadratically faster than classically expected amount considering the fact that the number of combinations is $O\left(\widetilde{N}^{2}\right)$ in our crossover procedure (see the next subsection for the details). As for the space complexity, we spend poly $(\log N)$ qubits and $O(\log N)$ classical bits as is easily evaluated from the description of Algorithm 2 (Fig. 22). 


\subsection{Comparison with a classical counterpart}

The classical counterpart of our algorithm is the one described in Fig. 7 ( Algorithm (3). As is obvious from its structure, it should have the same output and the same number of iterations as our algorithm with almost certainty as long as the same random seed is used for step 1 to construct $R$ for each value of $t$. The computational costs of individual procedures in Algorithm 3 are as

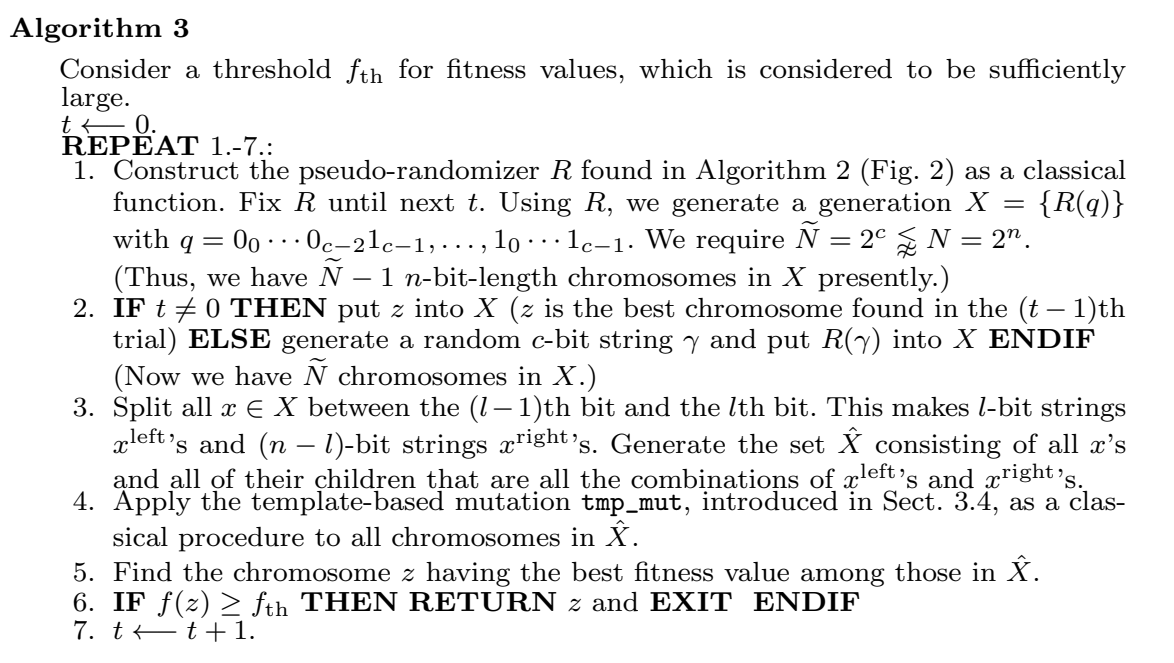

Fig. 7 Description of a classical counterpart of our algorithm.

follows.

- Generating $X$ in the steps 1.-2. takes $O(\widetilde{N}$ poly $(c n))$ basic operations.

- We need to use $O\left(n \widetilde{N}^{2}\right)$ space and $O\left(n \widetilde{N}^{2}\right)$ basic operations to perform all the crossovers among $\widetilde{N}$ parents.

- Mutations acting on the individuals of $\hat{X}$ take $O\left(n \tilde{N}^{2}\right)$ basic operations.

- The selection to find the best individual from $\hat{X}$ takes $O\left(\tilde{N}^{2}\right.$ poly $\left.(n)\right)$ basic operations considering the cost $\operatorname{poly}(n)$ of calculating a fitness value.

As $n=\log _{2} N$ and $c=\log _{2} \tilde{N}$, the time and space complexities are $O\left(\tilde{N}^{2}\right.$ poly $\left.(\log N)\right)$ and $O\left(\widetilde{N}^{2} \log N\right)$, respectively, for each $t$, i.e., for handling each generation.

In contrast, as we have seen in Sect.4.2 the time and space complexities of our quantum genetic algorithm (described in Fig. 2) are $O(\widetilde{N} \operatorname{poly}(\log \widetilde{N} \log N))$ and poly $(\log N)$, respectively, for each $t$. Therefore, neglecting the difference between poly $(\log \widetilde{N} \log N)$ and poly $(\log N)$, we have achieved a quadratic speedup over its classical counterpart together with an exponential reduction in space.

The classical counterpart has been constructed by keeping the one-byone correspondence with the quantum algorithm. Thus there is a possibility 
that a better classical algorithm with the same behavior exists. This is in fact the case for Algorithm 3 . For a fairer comparison, now we reform Algorithm 3 and reduce its space complexity. The algorithm described in Fig. 8 (Algorithm 4) has the same output and the same number of repetitions as Algorithm 3 while its space complexity is exponentially reduced. We use the same pseudo-randomizer construction and the same mutation procedure as before. Of course, we set $\widetilde{N}=2^{c}$ and $N=2^{n}$ for integers $c$ and $n$ satisfying $1 \leq c<n$. In this algorithm, $R$ is called $O\left(\widetilde{N}^{2}\right)$ times for each value of $t$. We

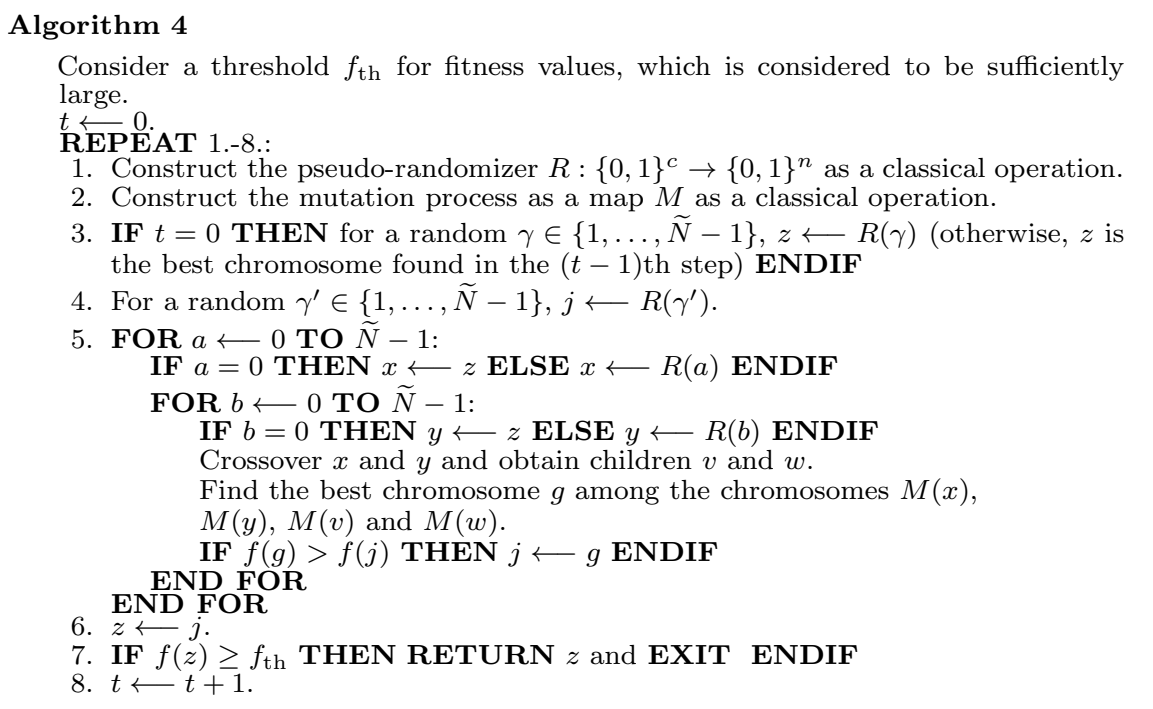

Fig. 8 Description of an improved classical counterpart of our algorithm.

know that $R$ internally takes poly $(\mathrm{cn})$ time. Therefore, this algorithm spends $O\left(\tilde{N}^{2}\right.$ poly $\left.(\log \tilde{N} \log N)\right)$ time for handling each generation. As for the space complexity, it spends only poly $(\log N)$ space, which is clear from the algorithm structure.

In comparison to this enhanced classical algorithm, our quantum algorithm still has a quadratically small time complexity as shown in Eq. (2).

\section{Discussion}

How to perform crossovers in a quantum manner was a pending problem in conventional quantum genetic algorithms [49,27,50,28]. In fact, a selective crossover for specific two chromosomes is expensive when they are component states of a superposition. Even if we attach address states pointing to the component states, we need to look up the classical data of the chromosomes to construct a quantum circuit realizing the unitary operation for this purpose, 
or more specifically, for placing address-controlled bit-flip gates appropriately. In our algorithm (Algorithm 2 shown in Fig. 2), we have avoided to mimic a classical way and chosen a different approach. We use two identical copies of a superposition corresponding to a generation and utilize relabelling of qubits so as to handle all possible combinations of substrings simultaneously. Obviously, the classical counterpart of our algorithm is the one that seeks for the best chromosome (after a mutation) among all possible crossovers for a chosen crossing site for each generation. Comparing our algorithm with the classical counterpart, we concluded that we have achieved a considerable reduction in the computational cost.

One may, however, claim that usually at most several crossovers are performed for a single generation in a classical genetic algorithm. Indeed, our algorithm is not aimed to be a quantum counterpart of a common classical genetic algorithm. As we discussed in Sect. 2, a straightforward conversion of a common classical algorithm into quantum one by simply incorporating a quantum search into the selection procedure has a problem: we need to either interpret nonconsecutive integers to consecutive ones or use an expensive construction for the inversion-about-average operation in order to perform the Grover-BBHT search, which causes a significant loss of performance. This problem should be resolved so as to find a meaningful quantum counterpart for the common case. Seemingly, the following workaround looks fine: (i) Use the pseudo-randomizer $R$ used in Algorithm2 instead of a random number generator to generate initial chromosomes of a generation. (ii) Apply a small number of crossovers. (iii) Use a selection procedure similar to that of Algorithm 2 Nevertheless, as we have discussed, it is not known how to construct the procedure (ii) as a unitary operation without the expensive process of looking up classical data of chromosomes. Therefore, it is the fact that a meaningful quantum counterpart is not easily found for a common classical genetic algorithm.

In view of the search space covered by each generation, a simultaneous crossover is, of course, desirable. Use of a superposition for this purpose was discussed 49,50 but not developed previously. In this sense, we have made a meaningful improvement by introducing Algorithm 2 .

Besides the crossover, let us discuss on the selection procedure. Our algorithm uses a variant of the Grover-BBHT search to achieve a quadratic speedup over its classical counterpart. The internal cost for each query is kept polynomial in the length of a chromosome because of the polynomial cost of our pseudo-randomizer, as described in Sect. 4. Apart from the complexity, there is some room to find a different design for the selection. Our algorithm is designed to carry over only the best chromosome $z$ to the next generation, among the chromosomes existing after the quantum crossover and mutation procedures. Since projective measurements are used in the quantum selection, it is inevitable to demolish other chromosomes. This can be a drawback because some of them may possess good fitness values albeit not the best. To mitigate this severe selection, one may keep the values of $z$ obtained in several elder generations as classical data. These values can be put into a later 
generation by modifying the register-initialization procedure slightly: one can modify the pseudo-randomizer so that it does not touch several input strings; then one can map them to the kept values of $z$. In this way, one may maintain a better diversity for high-fitness chromosomes. This is one possible extension of our algorithm.

There have not been many studies on quantum genetic algorithms so far. It is hoped that several or more different designs of genetic procedures will be developed for quantum computers.

\section{Summary}

We have proposed a genetic algorithm whose crossover, mutation and selection procedures have been all constructed as quantum routines so that quantum parallelism is effectively used. Its crossover procedure performs crossovers among all chromosomes of a generation. The run time of our algorithm to update each generation is quadratically faster than that of its classical counterpart, apart from negligible factors.

Acknowledgements A.S. is thankful to Shigeru Yamashita for his comment. A.S. and M.N. were supported by the "Open Research Center" Project for Private Universities: matching fund subsidy from MEXT. R.R. is supported by Industry Canada and CIFAR.

\section{A An example of constructing the pseudo-randomizer $R$}

In this appendix, we show an example to construct the pseudo-randomizer $R$ used in Algorithms 2 3 and 4. It should map a $c$-bit string to a pseudo-random $n$-bit string except for $0_{0} \cdots 0_{c-1}$ that is mapped to $0_{0} \cdots 0_{n-1}$. Its internal circuit complexity should be poly $(\mathrm{cn})$. As we use a quantum circuit to realize it in Algorithm 2 it is desirable to employ a circuit structure that is originally unitary.

Consider inputs $a \in\{0,1\}^{c}$. We design a circuit that maps $a_{0} \cdots a_{c-1} 0_{0} \cdots 0_{n-1}$ to $a_{0} \cdots a_{c-1} \kappa_{0} \cdots \kappa_{n-1}$ with $\kappa=R(a)$, an $n$-bit pseudo-random number (here, $a_{0} \cdots a_{c-1}$ and $\kappa_{0} \cdots \kappa_{n-1}$ are the binary representations of $a$ and $\left.\kappa\right)$. By the definition of $R$, the circuit preserves $0_{0} \cdots 0_{c-1} 0_{0} \cdots 0_{n-1}$. This circuit is generated by function gen_r_circ () described in Fig. 9 As is clear from the description, the circuit output from this function

FUNCTION gen_r_circ():

Comment: We use wires $v_{0}, \ldots, v_{c-1}, w_{0}, \ldots, w_{n-1}$.

FOR $i \longleftarrow 0$ TO $c-1$ :

(1) Use a random number generator to generate an $n$-bit integer $\gamma$. Write its binary representation as $\gamma_{0} \cdots \gamma_{n-1}$.

(2) Using the wire $v_{i}$ as the control wire (namely, the control bit), output the gate controlled- $\left(X_{0}\right)^{\gamma_{0}} \otimes \cdots \otimes\left(X_{n-1}\right)^{\gamma_{n-1}}$ with $X_{k}$ the bit flip gate acting on the wire $w_{k}(k=0, \ldots, n-1)$. In this gate, the bit flips are active under the condition that $v_{i}=1$

END FOR

Fig. 9 Description of function gen_r_circ () .

can be directly used as a quantum circuit. Using the circuit $C=$ gen_r_circ(), we have 
$|a\rangle|0\rangle \stackrel{C}{\mapsto}|a\rangle|R(a)\rangle \quad \forall a \in\{0,1\}^{c}$. The circuit complexity of $C$ is $O(c n)$ because, for each $i$, at most $n$ CNOT gates are used to decompose the gate output from step (2). In addition, gen_r_circ () spends $O(c$ poly $(n))$ time when a common random number generator 2131 . is used in step (1).

Note that the function gen_r_circ () is called only once for each $t$, in the beginning of step 1 in Algorithms 2 [3 and 4 We have only to reuse the circuit $C$ for the use of the pseudo-randomizer until $t$ is incremented.

It is expected that outputs from the circuit $C$ possess good uniformity if we use a good random number generator in step (1) of gen_r_circ() for generating $C$. Let us write $\gamma$ as $\gamma(i)$ to emphasize its dependence on $i$. For a nonzero input $a_{0} \cdots a_{c-1}$, the $k$ th bit of the output $R(a)$ is $\sum_{i=0}^{c-1} a_{i} \cdot \gamma_{k}(i) \bmod 2$. This indicates that, for two different inputs $a$ and $a^{\prime}$, the $k$ th bits of $R(a)$ and $R\left(a^{\prime}\right)$ differ with probability $1 / 2$ in the ideal case where $\gamma(i)$ 's are generated from a true random number generator. This is because $a$ and $a^{\prime}$ differ by at least a single bit. It also indicates that two different bits, the $k$ th and the $k^{\prime}$ th bits, of $R(a)$ for a nonzero input $a$ differ with the probability $1 / 2$ in the ideal case. This is because $\gamma_{k}(i)$ and $\gamma_{k^{\prime}}(i)$ differ with the probability $1 / 2$.

Now we show the result of our numerical test of $C$. We tried statistical tests of randomness 21,39 to test pseudo-random numbers output from $C$, using NIST's Statistical Test Suite (STS) (version 2.1.1) 39. We set $c=10$ and $n=32$. Mersenne Twister (MT) (version mt19937ar) 31 was used to generate $\gamma$ in step (1) of gen_r_circ () . We used the seed value 121212 and did not reset MT during the circuit generation. The circuit $C$ output from gen_r_circ () , of course, consisted of 10 outputs from step (2). For this $C$, we used the inputs $a \in\{0,1\}^{c} \backslash\left\{0_{0} \cdots 0_{c-1}\right\}$ from smaller to larger and obtained corresponding outputs $R(a)$ by numerical computation. We obtained $1023 \times 32$ bits in total in the outputs, since $2^{10}-1=1023$. We regarded them as a serial bit string from left to right and used STS in its default setting to test the string. In the execution of STS, we used 25 binary sequences with length 1200 as samples from the string. The following tests were tried with the default parameter values in STS: the Frequency Test, the Block Frequency Test, the Cumulative Sums Test, the Runs Test, the Longest-Run-of-Ones Test, the Binary Matrix Rank Test, the Spectral DFT Test and the Serial Test. The string passed the tests except for the Binary Matrix Rank Test. It should be noted that the input length was too small for the binary matrix rank test 39. In addition, randomness is not very strictly required for the use in evolutionary computing. Therefore, considering the tests that the string passed, we may claim that gen_r_circ() generates a usable pseudo-randomizer circuit for our algorithm.

We conducted another test: We generated ten circuits by calling gen_r_circ() ten times without resetting MT, using the seed value 676767 . For each circuit, we performed the same process as above to obtain the serial bit string. We obtained ten serial bit strings in total and tested the concatenated string using STS. As samples input to STS, we used 25 binary sequences with length 12000 . The concatenated string passed the tests except for the Binary Matrix Rank Test and the Spectral DFT Test. It was unexpected that it did not pass the spectral DFT test. It requires a further investigation to reveal the reason of this phenomenon.

The results of the first and the second tests are summarized in Table 1 In summary for this appendix, we found that a pseudo-randomizer circuit whose outputs possess enough randomness for the use in evolutionary computing can be generated by the function gen_r_circ () . It is hoped that the function will be improved so as to achieve better randomness for the sake of general use.

\section{References}

1. Ahuja, A., Kapoor, S.: A quantum algorithm for finding the maximum (1999). arXiv:quant-ph/9911082

2. Barnum, H., Bernstein, H.J., Spector, L.: A quantum circuit for OR (1999). arXiv:quant$\mathrm{ph} / 9907056$

3. Boyer, M., Brassard, G., Høyer, P., Tapp, A.: Tight bounds on quantum searching. Fortschr. Phys. 46, 493-505 (1998) 
Table 1 List of test results for the first and the second tests we performed (see the text for the details of the tests). Each value is a $P$-value (see Ref. 39 for its meaning for each test). The asterisk $*$ indicates a failure to pass the test. We should mention that the values for the Longest-Run-of-Ones Test accidentally coincided, while the internally-used results for sample sequences were different.

\begin{tabular}{|r|l|l|}
\hline & 1st Test & 2nd Test \\
\hline Frequency Test & 0.021262 & 0.186566 \\
Block Frequency Test & 0.105618 & 0.001156 \\
Cumulative Sums Test (Forward) & 0.105618 & 0.029796 \\
Cumulative Sums Test (Reverse) & 0.001691 & 0.057146 \\
Runs Test & 0.141256 & 0.010606 \\
Longest-Run-of-Ones Test & 0.875539 & 0.875539 \\
Binary Matrix Rank Test & $0.000000 *$ & $0.000000 *$ \\
Spectral DFT Test & 0.000533 & $0.000000 *$ \\
Serial Test & 0.041438 & 0.186566 \\
\hline
\end{tabular}

4. Brassard, G., Høyer, P., Tapp, A.: Quantum counting. In: K.G. Larsen, S. Skyum, G. Winskel (eds.) Proceedings of Automata, Languages and Programming, 25th International Colloquium (ICALP'98) (LNCS 1443), pp. 820-831. Aalborg, Denmark, 13-17 July 1998, Springer-Verlag, Berlin (1998). arXiv:quant-ph/9805082

5. Chakraborty, S., Radhakrishnan, J., Raghunathan, N.: Bounds for error reduction with few quantum queries. In: C. Chekuri, K. Jansen, J. Rolim, L. Trevisan (eds.) Proceedings of the 9th International Workshop on Randomization and Computation (RANDOM 2005) (LNCS 3624), pp. 245-256. Berkeley, CA, 22-24 August 2005, Springer-Verlag, Berlin (2005)

6. Chen, M., Quan, H.: Quantum-inspired evolutionary algorithm based on estimation of distribution. In: Proceedings of the 2nd International Conference on Bio-Inspired Computing: Theories and Applications (BIC-TA 2007), pp. 17-19. Zhengzhou, China, 14-17 September 2007, IEEE Press, Piscataway, NJ (2007)

7. Ding, S., Jin, Z., Yang, Q.: Evolving quantum oracles with hybrid quantum-inspired evolutionary algorithm (2006). arXiv:quant-ph/0610105

8. Dürr, C., Høyer, P.: A quantum algorithm for finding the minimum (1996). arXiv:quant$\mathrm{ph} / 9607014$

9. Gepp, A., Stocks, P.: A review of procedures to evolve quantum algorithms (2007) arXiv:0708.3278

10. Giraldi, G.A., Portugal, R., Thess, R.N.: Genetic algorithms and quantum computation (2004). arXiv:cs/0403003

11. Goldberg, D.E.: Genetic Algorithms in Search, Optimization, and Machine Learning. Addison-Wesley, Reading, MA (1989)

12. Grover, L.K.: A fast quantum mechanical algorithm for database search. In: Proceedings of the 28th Annual ACM Symposium on Theory of Computing (STOC 1996), pp. 212219. Philadelphia, PA, 22-24 May 1996, ACM Press, New York, NY (1996)

13. Grover, L.K.: Quantum search on structured problems (1998). arXiv:quant-ph/9802035

14. Grover, L.K.: Fixed-point quantum search. Phys. Rev. Lett. 95, 150501-1-4 (2005)

15. Gruska, J.: Quantum Computing. McGraw-Hill, London (1999)

16. Han, K.H., Kim, J.H.: Genetic quantum algorithm and its application to combinatorial optimization problem. In: Proceedings of the 2000 Congress on Evolutionary Computation (CEC2000), pp. 1354-1360. La Jolla, CA, 16-19 July 2000, IEEE Press, Piscataway, NJ (2000)

17. Han, K.H., Kim, J.H.: Quantum-inspired evolutionary algorithm for a class of combinatorial optimization. IEEE Trans. Evol. Comput. 6(6), 580-593 (2002)

18. Han, K.H., Kim, J.H.: Quantum-inspired evolutionary algorithms with a new termination criterion, $h_{\epsilon}$ gate, and two-phase scheme. IEEE Trans. Evol. Comput. 8(2), 156-169 (2004) 
19. Holland, J.H.: Adaptation in Natural and Artificial Systems: An Introductory Analysis with Applications to Biology, Control and Artificial Intelligence. The University of Michigan Press, Ann Arbor, MI (1975)

20. Johannsen, D., Kuru, P.P., Lengler, J.: Can quantum search accelerate evolutionary algorithms? In: M. Pelikan, J. Branke (eds.) Proceedings of the 12th Annual Genetic and Evolutionary Computation Conference (GECCO-2010), pp. 1433-1440. Portland, OR, 7-11 July 2010, ACM, New York, NY (2010)

21. Knuth, D.E.: The Art of Computer Programming, Vol. 2: Seminumerical Algorithms, 3rd Ed. Addison-Wesley, Reading, MA (1997). Chap. 3

22. Leier, A., Banzhaf, W.: Evolving Hogg's quantum algorithm using linear-tree GP. In: E. Cantú-Paz, J.A. Foster, K. Deb, L.D. Davis, R. Roy, U.M. O'Reilly, H.G. Beyer, R. Standish, G. Kendall, S. Wilson, M. Harman, J. Wegener, D. Dasgupta, M.A. Potter, A.C. Schultz, K.A. Dowsland, N. Jonoska, J. Miller (eds.) Proceedings of the Genetic and Evolutionary Computation Conference 2003 (GECCO-2003), Part I (LNCS 2723), pp. 390-400. Chicago, IL, 12-16 July 2003, Springer-Verlag, Berlin (2003)

23. Leier, A., Banzhaf, W.: Comparison of selection strategies for evolutionary quantum circuit design. In: K. Deb (ed.) Proceedings of the Genetic and Evolutionary Computation Conference 2004 (GECCO-2004), Part II (LNCS 3103), pp. 557-568. Seattle, WA, 26-30 June 2004, Springer-Verlag, Berlin (2004)

24. Liao, R., Wang, X., Qin, Z.: A novel quantum-inspired genetic algorithm with expanded solution space. In: Proceedings of the 2010 Second International Conference on Intelligent Human-Machine Systems and Cybernetics (IHMSC 2010), pp. 192-195. Nanjing, China, 26-28 August 2010, IEEE Computer Society, Los Alamitos, CA (2010)

25. Lukac, M., Perkowski, M.: Evolving quantum circuits using genetic algorithm. In: A. Stoica, D. Keymeulen, J. Lohn (eds.) Proceedings of the 2002 NASA/DoD Conference on Evolvable Hardware, pp. 177-181. Alexandria, VA, 15-18 July 2002, IEEE Computer Society, Los Alamitos, CA (2002)

26. Lukac, M., Perkowski, M., Goi, H., Pivtoraiko, M., Yu, C.H., Chung, K., Jee, H., Kim, B.G., Kim, Y.D.: Evolutionary approach to quantum and reversible circuits synthesis. In: S.N. Yanushkevich (ed.) Artificial Intelligence in Logic Design, pp. 201-257. Kluwer Academic Publisher, Dordrecht (2004)

27. Malossini, A., Blanzieri, E., Calarco, T.: QGA: quantum genetic algorithm (2004). Technical Report: \#DIT-04-105, Dec. 2004, Univ. Trento, http://www.dit.unitn.it

28. Malossini, A., Blanzieri, E., Calarco, T.: Quantum genetic optimization. IEEE Trans. Evol. Comput. 12(2), 231-241 (2008)

29. Massey, P., Clark, J.A., Stepney, S.: Evolving quantum circuits and programs through genetic programming. In: K. Deb (ed.) Proceedings of the Genetic and Evolutionary Computation Conference 2004 (GECCO-2004), Part II (LNCS 3103), pp. 569-580. Seattle, WA, 26-30 June 2004, Springer-Verlag, Berlin (2004)

30. Massey, P., Clark, J.A., Stepney, S.: Human-competitive evolution of quantum computing artefacts by genetic programming. Evol. Comput. 14(1), 21-40 (2006)

31. Matsumoto, M., Nishimura, T.: Mersenne Twister: a 623-dimensionally equidistributed uniform pseudorandom number generator. ACM Trans. Model. Comput. Sim. 8, 3-30 (1998). Http://www.math.sci.hiroshima-u.ac.jp/ ${ }^{\sim}$ m-mat/MT/mt.html

32. Mitchell, M.: An Introduction to Genetic Algorithms. MIT Press, Cambridge, MA (1996)

33. Mohammed, A.M., Elhefnawy, N.A., El-Sherbiny, M.M., Hadhoud, M.M.: Quantum crossover based quantum genetic algorithm for solving non-linear programming. In: Proceedings of the 8th International Conference on INFOrmatics and Systems (INFOS2012), pp. BIO-145-153. Cairo, Egypt, 14-16 May 2012, IEEE, Piscataway, NJ (2012)

34. Nakayama, S., Imabeppu, T., Ono, S.: Pair swap strategy in quantum-inspired evolutionary algorithm (2006). In the Late-breaking papers of the 2006 Genetic and Evolutionary Computation Conference (GECCO-2006), Seattle, WA, 8-12 July 2006

35. Nakayama, S., Imabeppu, T., Ono, S., Iimura, I.: Consideration on pair swap strategy in quantum-inspired evolutionary algorithm. IEICE Trans. Inf. Sys. J89-D(9), 2134-2139 (2006). In Japanese 
36. Narayanan, A., Moore, M.: Quantum-inspired genetic algorithms. In: Proceedings of the IEEE 3rd International Conference on Evolutionary Computation (ICEC96), pp. 61-66. Nagoya, Japan, 20-22 May 1996, IEEE Press, Piscataway, NJ (1996)

37. Nielsen, M.A., Chuang, I.L.: Quantum Computation and Quantum Information. Cambridge University Press, Cambridge (2000)

38. Rubinstein, B.I.P.: Evolving quantum circuits using genetic programming. In: Proceedings of the 2001 Congress on Evolutionary Computation (CEC2001), pp. 144-151. Seoul, Korea, 27-30 May 2001, IEEE Press, Piscataway, NJ (2001)

39. Rukhin, A., Soto, J., Nechvatal, J., Smid, M., Barker, E., Leigh, S., Levenson, M., Vangel, M., Banks, D., Heckert, A., Dray, J., Vo, S.: A statistical test suite for random and pseudorandom number generators for cryptographic applications (2010). NIST Special Publication 800-22, Revision 1a, http://csrc.nist.gov/groups/ST/toolkit/rng/index.html

40. Rylander, B., Soule, T., Foster, J., Alves-Foss, J.: Quantum evolutionary programming. In: L. Spector, E.D. Goodman, A. Wu, W.B. Langdon, H.M. Voigt, M. Gen, S. Sen, M. Dorigo, S. Pezeshk, M.H. Garzon, E. Burke (eds.) Proceedings of the Genetic and Evolutionary Computation Conference (GECCO-2001), pp. 1005-1011. San Francisco, CA, 7-11 July 2001, Morgan Kaufmann, San Francisco (2001)

41. Sofge, D.A.: Prospective algorithms for quantum evolutionary computation. In: P.D. Bruza, W. Lawless, K. van Rijsbergen, D.A. Sofge, B. Coecke, S. Clark (eds.) Proceedings of the 2nd Quantum Interaction Symposium (QI-2008), pp. 98-105. Oxford, UK, 26-28 March 2008, College Publications, London (2008). arXiv:0804.1133

42. Soklakov, A.N., Schack, R.: Efficient state preparation for a register of quantum bits. Phys. Rev. A 73, 012307-1-13 (2006)

43. Spector, L.: Automatic Quantum Computer Programming: A Genetic Programming Approach. Springer, New York (2004, Paperback Ed. 2007)

44. Spector, L., Barnum, H., Bernstein, H.: Genetic programming for quantum computers. In: J.R. Koza (ed.) Genetic Programming 1998: Proceedings of the Third Annual Conference (GP-98), pp. 365-374. Madison, WI, 22-25 July 1998, Morgan Kaufmann, San Francisco (1998)

45. Spector, L., Barnum, H., Bernstein, H., Swamy, N.: Finding a better-than-classical quantum AND/OR algorithm using genetic programming. In: Proceedings of the 1999 Congress on Evolutionary Computation (CEC1999), pp. 2239-2246. Washington, D.C., 6-9 July 1999, IEEE Press, Piscataway, NJ (1999)

46. Spector, L., Klein, J.: Machine invention of quantum computing circuits by means of genetic programming. AI EDAM 22, 275-283 (2008)

47. Tanaka, Y., Ichikawa, T., Tada-Umezaki, M., Ota, Y., Nakahara, M.: Quantum oracles in terms of universal gate set. Int. J. Quant. Inf. 9, 1363-1381 (2011)

48. Tulsi, T., Grover, L.K., Patel, A.: A new algorithm for fixed point quantum search. Quant. Inf. Comput. 6, 483-494 (2006)

49. Udrescu, M., Prodan, L., Vlăduţiu, M.: Grover's algorithm and the evolutionary approach of quantum computation (2004). ACSA Report, "Politehnica" University of Timisoara, 15 Oct. 2004, http://www.acsa.upt.ro/publications/index.htm

50. Udrescu, M., Prodan, L., Vlăduţiu, M.: Implementing quantum genetic algorithms: A solution based on Grover's algorithm. In: Proceedings of the 3rd Conference on Computing Frontiers, pp. 71-81. Ischia, Italy, 3-5 May 2006, ACM Press, New York (2006)

51. Ventura, D., Martinez, T.: Initializing the amplitude distribution of a quantum state. Found. Phys. Lett. 12, 547-559 (1999)

52. Williams, C.P., Gray, A.G.: Automated design of quantum circuits. In: C.P. Williams (ed.) Quantum Computing and Quantum Communications: First NASA International Conference (LNCS 1509), pp. 113-125. Palm Springs, CA, 17-20 February 1998, Springer-Verlag, Berlin (1999)

53. Yabuki, T., Iba, H.: Genetic algorithms for quantum circuit design-evolving a simpler teleportation circuit. In: L.D. Whitley, D.E. Goldberg, E. Cantú-Paz, L. Spector, I.C. Parmee, H.G. Beyer (eds.) Proceedings of the 2000 Genetic and Evolutionary Computation Conference (GECCO-2000), pp. 425-430. Las Vegas, NV, 8-12 July 2000, Morgan Kaufmann, San Francisco (2000) 
54. Zhang, G.: Quantum-inspired evolutionary algorithms: a survey and empirical study. J. Heuristics 17, 303-351 (2011) 